\title{
Asymptotic model for twisted bent ferromagnetic wires with electric current
}

\author{
Abdel Kader Al Sayed ${ }^{2}$, Gilles Carbou ${ }^{1}$ and Stéphane Labbé ${ }^{2}$ \\ ${ }^{1}$ CNRS / UNIV PAU \& PAYS ADOUR, IPRA, LABORATOIRE DE MATHEMATIQUES ET \\ DE LEURS APPLICATIONS DE PAU, UMR CNRS 5142, Avenue de l'Université - BP 1155, \\ 64013 PAU CEDEX, FRANCE \\ ${ }^{2}$ Univ. Grenoble Alpes, CNRS, Grenoble INP*, LJK, 38000 Grenoble, France \\ * Institute of Engineering Univ. Grenoble Alpes \\ email:abdel-kader.al-sayed@univ-grenoble-alpes.fr, gilles.carbou@univ-pau.fr, \\ stephane.labbe@univ-grenoble-alpes.fr
}

\begin{abstract}
In this paper, we derive a one-dimensional asymptotic model for the dynamics of the magnetic moment in a twisted ferromagnetic nanowire with arbitrary elliptical cross-section, curvature and torsion.
\end{abstract}

Keywords: ferromagnetism, Landau-Lifschitz equation, nanowire, asymptotic process.

MSC: 35K55, 35Q60

\section{Introduction}

Ferromagnetic nanowires have promising potential applications in data storage devices (see [11]) and magnetic logic gates (see [1]). The wires used in these fields have complex shapes, with bends, notches or junctions. The study of domain wall formation and dynamics in such wires with complex geometry is crucial for applications (see $[1,11,12,13,14,16,19]$ ).

In this paper, we justify by asymptotic process a time-dependent one-dimensional model for a twisted, bent nanowire of variable cross-section. Our model takes into account the current effects.

Several works address the justification of $1 \mathrm{~d}$ models for ferromagnetic nanowires (see [6], [15], [7]), however they consider a constant shaped cross-section or a cross-section oriented by the normal and binormal vectors of the curve modeling the wire central line. In this work we are able to consider here narrowing zones (arising in wires with notches) and twist shaped wires.

First, recall the $3 \mathrm{~d}$ model for ferromagnetic materials (see $[5,10]$ ). We consider a ferromagnetic body occupying the volume $\Omega \subset \mathbb{R}^{3}$. We denote by $\mathbf{M}(\mathbf{t}, \mathbf{x})$ the magnetization distribution at the time $\mathbf{t}$ and at the point $\mathbf{x} \in \Omega$. At low temperature, the material satisfies the saturation constraint:

$$
|\mathbf{M}(\mathbf{t}, \mathbf{x})|=M_{s},
$$

where $M_{s}$ is independent of $\mathbf{t}$ and $\mathbf{x}$. The variations of $\mathbf{M}$ satisfy the following Landau-LifschitzGilbert equation:

$$
\frac{\partial \mathbf{M}}{\partial \mathbf{t}}-\frac{\alpha}{M_{s}} \mathbf{M} \times \frac{\partial \mathbf{M}}{\partial \mathbf{t}}=-\gamma \mathbf{M} \times H_{\text {eff }}-(\overrightarrow{\mathbf{u}} \cdot \nabla) \mathbf{M},
$$

where $\times$ is the cross product in $\mathbb{R}^{3}, \gamma$ is the gyromagnetic constant, $\alpha$ is the damping coefficient. The effective field $H_{\text {eff }}$ is given by:

$$
H_{\text {eff }}=\frac{A}{\mu_{0} M_{s}^{2}} \Delta \mathbf{M}+\mathbf{H}_{d}(\mathbf{M})+H_{a},
$$

where $A$ is the exchange constant, $\mu_{0}$ is the permeability of the vacuum, $H_{a}$ is the applied magnetic field and $\mathbf{H}_{d}(\mathbf{M})$ is the demagnetizing field that deduced from $\mathbf{M}$ by the operator $\mathbf{H}_{d}$ given by:

$$
\operatorname{curl} \mathbf{H}_{d}(\mathbf{M})=0 \quad \text { and } \quad \operatorname{div}\left(\mathbf{H}_{d}(\mathbf{M})+\overline{\mathbf{M}}\right)=0,
$$

where $\overline{\mathbf{M}}(\mathbf{t}, \mathbf{x})=\mathbf{M}(\mathbf{t}, \mathbf{x})$ for $\mathbf{x} \in \Omega$ and zero outside $\Omega$. 
The right-hand-side transport term in (1.2) describes the current effects: the velocity $\overrightarrow{\mathbf{u}}$ is given by

$$
\overrightarrow{\mathbf{u}}=\frac{p g \mu_{B}}{2 e M_{s}} \vec{J}
$$

where $\vec{J}$ is the current density, $p, g, \mu_{B}$ and $e$ are respectively the current polarization, the Landé factor, the Bohr Magneton and the electron charge (see [4] and [17]).

Writing $\mathbf{M}(\mathbf{t}, \mathbf{x})=M_{s} \mathbf{m}\left(\gamma M_{s} \mathbf{t}, \mathbf{x}\right), H_{a}(\mathbf{t}, \mathbf{x})=M_{s} \mathbf{h}_{a}\left(\gamma M_{s} \mathbf{t}, \mathbf{x}\right)$, and $\overrightarrow{\mathbf{u}}(\mathbf{t}, \mathbf{x})=\gamma M_{s} \overrightarrow{\mathbf{v}}\left(\gamma M_{s} \mathbf{t}, \mathbf{x}\right)$, we obtain the rescaled model:

$$
\left\{\begin{array}{l}
\partial_{t} \mathbf{m}-\alpha \mathbf{m} \times \partial_{t} \mathbf{m}=-\mathbf{m} \times \mathbf{h}_{\mathrm{eff}}-(\overrightarrow{\mathbf{v}} \cdot \nabla) \mathbf{m}, \\
\mathbf{h}_{\mathrm{eff}}=\ell^{2} \Delta \mathbf{m}+\mathbf{H}_{d}(\mathbf{m})+\mathbf{h}_{a} .
\end{array}\right.
$$

where the dimensionless time is $t=\gamma M_{s} \mathbf{t}$ and $\ell^{2}=\frac{A}{\mu_{0} M_{s}^{2}}$.

We consider a curved ferromagnetic wire with non constant elliptical cross-section. We introduce $\mathbf{s} \mapsto \Gamma(\mathbf{s})$, the arc-length parametrization of the wire central line. We assume that for all $\mathbf{s}$, the crosssection is an ellipse whose axis are directed by $\overrightarrow{\mathbf{e}}_{\mathbf{a}}(\mathbf{s})$ and $\overrightarrow{\mathbf{e}}_{\mathbf{b}}(\mathbf{s})$, where for all $\mathbf{s},\left(\Gamma^{\prime}(\mathbf{s}), \overrightarrow{\mathbf{e}}_{\mathbf{a}}(\mathbf{s}), \overrightarrow{\mathbf{e}}_{\mathbf{b}}(\mathbf{s})\right)$ forms a direct orthonormal basis of $\mathbb{R}^{3}$. We denote by $\eta \mathbf{a}(\mathbf{s})$ and $\eta \mathbf{b}(\mathbf{s})$ the associated semi-axis, where $\eta$ is a small dimensionless parameter. Therefore, the wire is parametrized by:

$$
\mathbf{x}=\Psi_{\eta}(\mathbf{s}, u, v):=\Gamma(\mathbf{s})+\eta\left(u \mathbf{a}(\mathbf{s}) \overrightarrow{\mathbf{e}}_{\mathbf{a}}(\mathbf{s})+v \mathbf{b}(\mathbf{s}) \overrightarrow{\mathbf{e}}_{\mathbf{b}}(\mathbf{s})\right),
$$

where $(\mathbf{s}, u, v) \in \mathcal{O}:=[0, L] \times B_{2}(0,1)$. We denote by $B_{2}(0,1)$ the unit ball of $\mathbb{R}^{2}$ centered at 0 and radius 1 and by $\Omega_{\eta}=\Psi_{\eta}(\mathcal{O})$ the domain occupied by the ferromagnetic wire presented in figure 1 .

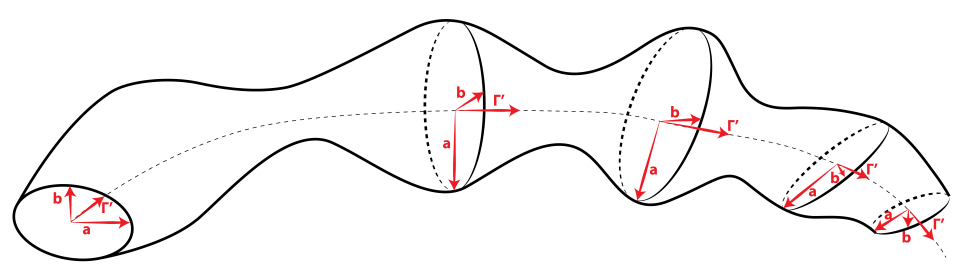

Figure 1: Ferromagnetic domain

We assume that $\Gamma \in \mathcal{C}^{2}([0, L]), \overrightarrow{\mathbf{e}_{\mathbf{a}}}$ and $\overrightarrow{\mathbf{e}_{\mathbf{b}}}$ are in $\mathcal{C}^{1}([0, L])$. We assume also that $\mathbf{a}$ and $\mathbf{b}$ are $\mathcal{C}^{1}$ on $[0, L]$ and bounded by below by a non negative constant.

Several Works study physically the effect of the geometrical form of the ferromagnetic wires on the magnetic moment behavior $[18,13,8]$. They study the effect of several geometrical aspects on the domain wall propagation. Respectively, they study the effect of the curvature, the torsion and the turning of the ribbon around its central wire on the domain wall propagation. All of these geometrical aspects are a particular cases of our geometry.

The existence of a global in time weak solution for (1.2) in $\Omega_{\eta}$ is established in [2] and [3]:

Proposition 1.1. We fix $\eta>0$. Let $\mathbf{m}_{0}^{\eta} \in H^{1}\left(\Omega_{\eta} ; \mathbb{R}^{3}\right)$ satisfying $\left|\mathbf{m}_{0}^{\eta}\right|=1$ a.e. Let $\mathbf{h}_{a, \eta} \in$ $\mathcal{C}_{b}^{0}\left(\mathbb{R}^{+} ; L^{2}\left(\Omega_{\eta}\right)\right)$ and $\overrightarrow{\mathbf{v}}_{\eta} \in \mathcal{C}_{b}^{0}\left(\mathbb{R}^{+} ; L^{\infty}\left(\Omega_{\eta}\right)\right)$. There exists $\mathbf{m}^{\eta}: \mathbb{R}^{+} \times \Omega_{\eta} \longrightarrow \mathbb{R}^{3}$ satisfying the saturation constraint $\left|\mathbf{m}^{\eta}(t, \mathbf{x})\right|=1$ a.e. such that

$$
\text { - for all } T \geq 0, \mathbf{m}^{\eta} \in L^{\infty}\left(0, T ; H^{1}\left(\Omega_{\eta}\right)\right) \text { and } \frac{\partial \mathbf{m}^{\eta}}{\partial t} \in L^{2}\left([0, T] \times \Omega_{\eta}\right) \text {, }
$$


- $\mathbf{m}^{\eta}(0, \cdot)=\mathbf{m}_{0}^{\eta}(\cdot)$ in the trace sense,

- For all $\Phi \in \mathbf{C}_{c}^{\infty}\left(\mathbb{R}^{+} ; H^{1}\left(\Omega_{\eta} ; \mathbb{R}^{3}\right)\right)$,

$$
\begin{aligned}
\int_{\mathbb{R}^{+} \times \Omega_{\eta}}\left(\frac{\partial \mathbf{m}^{\eta}}{\partial t}\right. & \left.-\alpha \mathbf{m}^{\eta} \times \frac{\partial \mathbf{m}^{\eta}}{\partial t}\right) \cdot \Phi d t d \mathbf{x}=\int_{\mathbb{R}^{+} \times \Omega_{\eta}} \sum_{i=1}^{3} \ell^{2}\left(\mathbf{m}^{\eta} \times \frac{\partial \mathbf{m}^{\eta}}{\partial \mathbf{x}_{i}}\right) \frac{\partial \Phi}{\partial \mathbf{x}_{i}} d t d \mathbf{x} \\
& -\int_{\mathbb{R}^{+} \times \Omega_{\eta}}\left(\mathbf{H}_{d}\left(\mathbf{m}^{\eta}\right)+\mathbf{h}_{a, \eta}\right) \cdot \Phi d t d \mathbf{x}-\int_{\mathbb{R}^{+} \times \Omega_{\eta}}\left(\overrightarrow{\mathbf{v}}_{\eta} \cdot \nabla\right) \mathbf{m}^{\eta} \cdot \Phi d t d \mathbf{x},
\end{aligned}
$$

- for almost every $t \geq 0$,

$$
\mathcal{E}_{\eta}\left(\mathbf{m}^{\eta}\right)(t)+\frac{\alpha}{2} \int_{0}^{t} \int_{\Omega_{\eta}}\left|\frac{\partial \mathbf{m}^{\eta}}{\partial t}\right|^{2} d t d \mathbf{x} \leq \mathcal{G}_{\eta}(t)\left(1+\mathcal{V}_{\eta}(t) \exp \int_{0}^{t} \mathcal{V}_{\eta}(t)(s) d s\right)
$$

where

$$
\mathcal{E}_{\eta}\left(\mathbf{m}^{\eta}\right)=\frac{\ell^{2}}{2} \int_{\Omega_{\eta}}\left|\nabla \mathbf{m}^{\eta}\right|^{2} d \mathbf{x}+\frac{1}{2} \int_{\mathbb{R}^{3}}\left|\mathbf{H}_{d}\left(\mathbf{m}^{\eta}\right)\right|^{2} d \mathbf{x}
$$

with

$$
\mathcal{G}_{\eta}(t)=\mathcal{E}\left(\mathbf{m}_{0}^{\eta}\right)+\int_{0}^{t}\left\|\mathbf{h}_{a, \eta}(\tau)\right\|_{L^{2}\left(\Omega_{\eta}\right)}^{2} d \tau \quad \text { and } \quad \mathcal{V}_{\eta}(t)(t)=\frac{2}{\alpha \ell^{2}} \int_{0}^{t}\left\|\overrightarrow{\mathbf{v}}_{\eta}(\tau)\right\|_{L^{\infty}\left(\Omega_{\eta}\right)}^{2} d \tau
$$

We aim to obtain an asymptotic one-dimensional model for the wire when the parameter $\eta$ tends to zero. By specifying the applied field and the electric current. We fix $h_{a} \in \mathcal{C}^{0}\left(\mathbb{R}^{+} ; L^{2}\left([0, L] ; \mathbb{R}^{3}\right)\right)$ and $j \in \mathcal{C}^{0}\left(\mathbb{R}^{+} ; L^{\infty}([0, L])\right)$. We assume that the applied field is constant in the cross-section and that the current is constant in the cross-section and oriented in the direction of the wire, i.e.

$$
\left\{\begin{array}{l}
\mathbf{h}_{a, \eta}\left(t, \Psi_{\eta}(\mathbf{s}, u, v)\right)=h_{a}(t, \mathbf{s}) \\
\overrightarrow{\mathbf{v}}_{\eta}\left(t, \Psi_{\eta}(\mathbf{s}, u, v)\right)=j(t, \mathbf{s}) \Gamma^{\prime}(\mathbf{s}) .
\end{array}\right.
$$

Then, we obtain the following theorem:

Theorem 1.1. Let $\mathbf{h}_{a, \eta} \in \mathcal{C}_{b}^{0}\left(\mathbb{R}^{+} ; L^{2}\left(\Omega_{\eta}\right)\right), \overrightarrow{\mathbf{v}}_{\eta} \in \mathcal{C}_{b}^{0}\left(\mathbb{R}^{+} ; L^{\infty}\left(\Omega_{\eta}\right)\right)$ defined by (1.10). Let $m_{0} \in$ $H^{1}\left([0, L] ; S^{2}\right)$. For $\eta>0$, we define the initial data $\mathbf{m}_{0}^{\eta} \in H^{1}\left(\Omega_{\eta} ; S^{2}\right)$ by: $\mathbf{m}_{0}^{\eta}\left(\Psi_{\eta}(\mathbf{s}, u, v)\right)=m_{0}(\mathbf{s})$.

We introduce the weak solution $\mathbf{m}^{\eta}$ of (1.2) given by Proposition 1.1 with initial data $\mathbf{m}_{0}^{\eta}$. We define $m^{\eta}: \mathbb{R}^{+} \times \mathcal{O} \longrightarrow S^{2}$ by

$$
m^{\eta}(t, \mathbf{s}, u, v)=\mathbf{m}^{\eta}\left(t, \Psi_{\eta}(\mathbf{s}, u, v)\right) .
$$

Then when $\eta$ tends to zero, there exists a subsequence still denoted by $m^{\eta}$ such that $m^{\eta} \rightarrow m$ in $L^{\infty}\left(0, T ; H^{1}(\mathcal{O})\right)$ weak *. In addition, $m$ does not depend on $u$ and $v$ and satisfies:

- $|m(t, \mathbf{s})|=1$ a.e., $\partial_{\mathbf{s}} m \in L^{\infty}\left([0, T] ; L^{2}([0, L])\right)$ and $\partial_{t} m \in L^{2}([0, T] \times[0, L])$ for all $T$,

- $m(0, \mathbf{s})=m_{0}(\mathbf{s})$ in the trace sense,

- for all $\phi \in \mathbf{C}_{c}^{\infty}\left(\mathbb{R}^{+} ; H^{1}([0, L])\right)$,

$$
\begin{aligned}
\int_{\mathbb{R}^{+} \times[0, L]} \sigma\left(\frac{\partial m}{\partial t}-\alpha m \times \frac{\partial m}{\partial t}\right) \cdot \phi d t d \mathbf{s}=\int_{\mathbb{R}^{+} \times[0, L]} \sigma \ell^{2}\left(m \times \frac{\partial m}{\partial \mathbf{s}}\right) \cdot \frac{\partial \phi}{\partial \mathbf{s}} d t d \mathbf{s} \\
-\int_{\mathbb{R}^{+\times[0, L]}} \sigma\left(H_{d}(m)+h_{a}\right) \cdot \Psi d t d \mathbf{s}-\int_{\mathbb{R}^{+} \times[0, L]} \sigma j \partial_{\mathbf{s}} m \cdot \Phi d t d \mathbf{s}
\end{aligned}
$$

where $\sigma(\mathbf{s})=\pi \mathbf{a}(\mathbf{s}) \mathbf{b}(\mathbf{s})$ and where the resulting demagnetizing field $H_{d}(m)$ is given by:

$$
H_{d}(m)=-\frac{\mathbf{b}}{\mathbf{a}+\mathbf{b}}\left(m \cdot \overrightarrow{\mathbf{e}}_{\mathbf{a}}\right) \overrightarrow{\mathbf{e}}_{\mathbf{a}}-\frac{\mathbf{a}}{\mathbf{a}+\mathbf{b}}\left(m \cdot \overrightarrow{\mathbf{e}}_{\mathbf{b}}\right) \overrightarrow{\mathbf{e}}_{\mathbf{b}}
$$


Remark 1.2. From the physical point of view $\overrightarrow{\mathbf{u}}$ is proportional to the density current $J$ (see (1.5)), it is natural to assume that the flux of $\overrightarrow{\mathbf{v}}_{\eta}$ through the cross-section is constant along the wire. For the asymptotic model, the corresponding assumption is that $\mathbf{s} \mapsto \sigma(\mathbf{s}) j(\mathbf{s})$ is constant along the wire.

Our one dimensional model is equivalent to smooth solutions of the model:

$$
\frac{\partial m}{\partial t}-\alpha m \times \frac{\partial m}{\partial t}=-m \times\left(\ell^{2} \frac{\partial^{2} m}{\partial \mathbf{s}^{2}}+\ell^{2} \frac{\sigma^{\prime}}{\sigma} \frac{\partial m}{\partial \mathbf{s}}+H_{d}(m)+h_{a}\right)-j \partial_{\mathbf{s}} m .
$$

The localization of the demagnetizing field in (1.11) has been already observed in [6] and [15].

It can be more convenient to describe $m$ in the mobile frame $\left(\Gamma^{\prime}(\mathbf{s}), \overrightarrow{\mathbf{e}}_{\mathbf{a}}(\mathbf{s}), \overrightarrow{\mathbf{e}}_{\mathbf{b}}(\mathbf{s})\right)$. For this purpose, we introduce $r_{1}, r_{2}, r_{3}$ in $\mathcal{C}^{0}([0, L])$ such that:

$$
\left\{\begin{array}{l}
\Gamma^{\prime \prime}(\mathbf{s})=r_{3}(\mathbf{s}) \overrightarrow{\mathbf{e}_{\mathbf{a}}}(\mathbf{s})-r_{2}(\mathbf{s}) \overrightarrow{\mathbf{e}_{\mathbf{b}}}(\mathbf{s}) \\
\frac{d \overrightarrow{\mathbf{e}_{\mathbf{a}}}}{d \mathbf{s}}(\mathbf{s})=-r_{3}(\mathbf{s}) \Gamma^{\prime}(\mathbf{s})+r_{1}(\mathbf{s}) \overrightarrow{\mathbf{e}_{\mathbf{b}}}(\mathbf{s}), \\
\frac{d \overrightarrow{\mathbf{e}_{\mathbf{b}}}}{d \mathbf{s}}(\mathbf{s})=r_{2}(\mathbf{s}) \Gamma^{\prime}(\mathbf{s})-r_{1}(\mathbf{s}) \overrightarrow{\mathbf{e}_{\mathbf{a}}}(\mathbf{s})
\end{array}\right.
$$

We denote by $R(\mathbf{s})=\left(\begin{array}{c}r_{1}(\mathbf{s}) \\ r_{2}(\mathbf{s}) \\ \left.r_{3}(\mathbf{s})\right)\end{array}\right)$ and by $\mathrm{m}=\left(\begin{array}{c}\mathrm{m}_{1} \\ \mathrm{~m}_{2} \\ \mathrm{~m}_{3}\end{array}\right)$ the coordinates of $m$ in the mobile frame:

$$
m(t, \mathbf{s})=\mathrm{m}_{1}(t, \mathbf{s}) \Gamma^{\prime}(\mathbf{s})+\mathrm{m}_{2}(t, \mathbf{s}) \overrightarrow{\mathbf{e}}_{\mathbf{a}}(\mathbf{s})+\mathrm{m}_{3}(t, \mathbf{s}) \overrightarrow{\mathbf{e}}_{\mathbf{b}}(\mathbf{s}) .
$$

We have $|\mathrm{m}|=1$ for all $t$ and $\mathbf{s}$, and $m$ satisfies (1.12) if and only if $\mathrm{m}$ satisfies:

$$
\frac{\partial \mathrm{m}}{\partial t}-\alpha \mathrm{m} \times \frac{\partial \mathrm{m}}{\partial t}=-\mathrm{m} \times \mathcal{H}(\mathrm{m})-j\left(\partial_{\mathrm{s}} \mathrm{m}+R \times \mathrm{m}\right),
$$

with

$$
\begin{aligned}
\mathcal{H}(\mathrm{m})= & \ell^{2} \frac{\partial^{2} \mathrm{~m}}{\partial \mathbf{s}^{2}}+2 \ell^{2} R \times \frac{\partial \mathrm{m}}{\partial \mathbf{s}}+\ell^{2} \frac{d R}{d \mathbf{s}} \times \mathrm{m}+\ell^{2} R \times(R \times \mathrm{m})+\ell^{2} \frac{\sigma^{\prime}}{\sigma}\left(\frac{\partial \mathrm{m}}{\partial \mathbf{s}}+R \times \mathrm{m}\right) \\
& -\frac{\mathbf{b}}{\mathbf{a}+\mathbf{b}} \mathrm{m}_{2}-\frac{\mathbf{a}}{\mathbf{a}+\mathbf{b}} \mathrm{m}_{3}+\mathrm{h}_{a},
\end{aligned}
$$

where $\mathrm{h}_{a}$ are the coordinates of $h_{a}$ in the mobile frame.

The paper is organized as follows. First, we achieve uniform bounds for $m^{\eta}$ by writing the energy formula (1.9) using the new variables $(\mathbf{s}, u, v)$ in Section 2. Second, we take the limit of the formulation verified by $m^{\eta}$ as $\eta$ tends to zero in Section 3. To achieve this, we utilize the uniform bounds achieved in the preceding section and rewrite the weak formulation 1.8 in the new variables. Finally, the limit of the demagnetizing field is characterized in Section 4.

\section{Uniform Estimates for the Rescaled Formulation}

In this section, we aim to obtain uniform bounds for $m^{\eta}$, by rewriting the energy formula (1.9) in the variables $(t, \mathbf{s}, u, v)$.

We compute the differential of $\Psi_{\eta}: \mathcal{O} \longrightarrow \Omega_{\eta}$ given by (1.7) with respect to its variables and using (1.13), we obtain that:

$$
\begin{aligned}
\frac{\partial \Psi_{\eta}}{\partial \mathbf{s}} & =\left(1-\eta\left(u \mathbf{a}(\mathbf{s}) r_{1}-v \mathbf{b}(\mathbf{s}) r_{2}\right)\right) \Gamma^{\prime}(\mathbf{s})+\eta\left(u \mathbf{a}^{\prime}(\mathbf{s})-v \mathbf{b}(\mathbf{s}) r_{3}\right) \overrightarrow{\mathbf{e}}_{\mathbf{a}}+\eta\left(u \mathbf{a}(\mathbf{s}) r_{3}+v \mathbf{b}^{\prime}(\mathbf{s})\right) \overrightarrow{\mathbf{e}}_{\mathbf{b}} \\
& =: \Gamma^{\prime}(s)\left(1+\eta g_{1}\right)+\eta g_{2} \overrightarrow{\mathbf{e}}_{\mathbf{a}}+\eta g_{3} \overrightarrow{\mathbf{e}}_{\mathbf{b}},
\end{aligned}
$$


where

$$
\begin{aligned}
& g_{1}(\mathbf{s}, u, v)=-u \mathbf{a}(\mathbf{s}) r_{1}(\mathbf{s})-v \mathbf{b}(\mathbf{s}) r_{2}(\mathbf{s}), \\
& g_{2}(\mathbf{s}, u, v)=u \mathbf{a}^{\prime}(\mathbf{s})-v \mathbf{b}(\mathbf{s}) r_{3}(\mathbf{s}), \\
& g_{3}(\mathbf{s}, u, v)=v \mathbf{b}^{\prime}(\mathbf{s})+u \mathbf{a}(\mathbf{s}) r_{3}(\mathbf{s}) .
\end{aligned}
$$

Furthermore, we have

$$
\frac{\partial \Psi}{\partial u}=\eta \mathbf{a}(\mathbf{s}) \overrightarrow{\mathbf{e}}_{\mathbf{a}} \quad \text { and } \quad \frac{\partial \Psi}{\partial v}=\eta \mathbf{b}(\mathbf{s}) \overrightarrow{\mathbf{e}}_{\mathbf{b}} .
$$

Thus, we obtain:

$$
\frac{\partial \Psi_{\eta}}{\partial \mathbf{s}}=\Gamma^{\prime}(\mathbf{s})\left(1+\eta g_{1}\right)+\frac{g_{2}}{\mathbf{a}(\mathbf{s})} \frac{\partial \Psi_{\eta}}{\partial u}+\frac{g_{3}}{\mathbf{b}(\mathbf{s})} \frac{\partial \Psi_{\eta}}{\partial v} .
$$

Then,

$$
\Gamma^{\prime}(\mathbf{s})=\frac{1}{1+\eta g_{1}}\left(\frac{\partial \Psi_{\eta}}{\partial \mathbf{s}}-\frac{g_{2}}{\mathbf{a}} \frac{\partial \Psi_{\eta}}{\partial u}-\frac{g_{3}}{\mathbf{b}} \frac{\partial \Psi_{\eta}}{\partial v}\right), \quad \overrightarrow{\mathbf{e}}_{\mathbf{a}}=\frac{1}{\eta \mathbf{a}} \frac{\partial \Psi_{\eta}}{\partial u} \quad \text { and } \quad \overrightarrow{\mathbf{e}}_{\mathbf{b}}=\frac{1}{\eta \mathbf{b}} \frac{\partial \Psi_{\eta}}{\partial v} .
$$

The Jacobian determinant of $\Psi_{\eta}$ is given by:

$$
J(s, u, v)=\eta^{2}\left(1+\eta g_{1}(s, u, v)\right) \mathbf{a}(\mathbf{s}) \mathbf{b}(\mathbf{s}),
$$

so that by changing the variable formula, for $f: \Omega_{\eta} \longrightarrow \mathbb{R}$ or $\mathbb{R}^{3}$, we have:

$$
\int_{\Omega_{\eta}} f(\mathbf{x}) d \mathbf{x}=\eta^{2} \int_{\mathcal{O}} \mathbf{a}(\mathbf{s}) \mathbf{b}(\mathbf{s}) f\left(\Psi_{\eta}(\mathbf{s}, u, v)\right)\left(1+\eta g_{1}(\mathbf{s}, u, v)\right) d \mathbf{s} d u d v .
$$

In order to estimate $\left\|\nabla \mathbf{m}^{\eta}\right\|_{L^{2}\left(\Omega^{\eta}\right)}^{2}$, we remark that whatever the orthonormal frame $\left(\vec{\xi}_{1}, \vec{\xi}_{2}, \vec{\xi}_{3}\right)$,

$$
\left|\nabla \mathbf{m}^{\eta}(\mathbf{x})\right|^{2}=\sum_{i=1}^{3}\left|d \mathbf{m}^{\eta}(\mathbf{x})\left(\xi_{i}\right)\right|^{2} .
$$

With the orthonormal frame $\left(\Gamma^{\prime}(\mathbf{s}), \overrightarrow{\mathbf{e}}_{\mathbf{a}}(\mathbf{s}), \overrightarrow{\mathbf{e}}_{\mathbf{b}}(\mathbf{s})\right)$, we obtain that

$$
\begin{aligned}
\left|\nabla \mathbf{m}^{\eta}\left(\Psi_{\eta}(\mathbf{s}, u, v)\right)\right|^{2}= & \left|d \mathbf{m}^{\eta}\left(\Psi_{\eta}(\mathbf{s}, u, v)\right)\left(\Gamma^{\prime}(\mathbf{s})\right)\right|^{2}+\left|d \mathbf{m}^{\eta}\left(\Psi_{\eta}(\mathbf{s}, u, v)\right)\left(\overrightarrow{\mathbf{e}}_{\mathbf{a}}(\mathbf{s})\right)\right|^{2} \\
& +\left|d \mathbf{m}^{\eta}\left(\Psi_{\eta}(\mathbf{s}, u, v)\right)\left(\overrightarrow{\mathbf{e}}_{\mathbf{b}}(\mathbf{s})\right)\right|^{2} .
\end{aligned}
$$

Since $m^{\eta}=\mathbf{m}^{\eta} \circ \Psi_{\eta}$, then using the chain rule, and (2.3), we obtain that:

$$
\begin{aligned}
& d \mathbf{m}^{\eta}\left(\Psi_{\eta}(\mathbf{s}, u, v)\right)\left(\Gamma^{\prime}(\mathbf{s})\right)=\frac{1}{1+\eta g_{1}}\left(\frac{\partial m^{\eta}}{\partial \mathbf{s}}-\frac{g_{2}}{\mathbf{a}} \frac{\partial m^{\eta}}{\partial u}-\frac{g_{3}}{\mathbf{b}} \frac{\partial m^{\eta}}{\partial v}\right), \\
& d \mathbf{m}^{\eta}\left(\Psi_{\eta}(\mathbf{s}, u, v)\right)\left(\overrightarrow{\mathbf{e}_{\mathbf{a}}}\right)=\frac{1}{\eta \mathbf{a}} \frac{\partial m^{\eta}}{\partial u}, \\
& d \mathbf{m}^{\eta}\left(\Psi_{\eta}(\mathbf{s}, u, v)\right)\left(\overrightarrow{\mathbf{e}_{\mathbf{b}}}\right)=\frac{1}{\eta \mathbf{b}} \frac{\partial m^{\eta}}{\partial v} .
\end{aligned}
$$

Thus, we have by (2.4) :

$$
\begin{aligned}
\int_{\Omega_{\eta}}\left|\nabla \mathbf{m}^{\eta}\right|^{2} d \mathbf{x}= & \eta^{2} \int_{\mathcal{O}} \frac{\mathbf{a b}}{1+\eta g_{1}}\left|\frac{\partial m^{\eta}}{\partial \mathbf{s}}-\frac{g_{2}}{\mathbf{a}} \frac{\partial m^{\eta}}{\partial u}-\frac{g_{3}}{\mathbf{b}} \frac{\partial m^{\eta}}{\partial v}\right|^{2} d \mathbf{s} d u d v \\
& +\int_{\mathcal{O}} \mathbf{a b}\left(1+\eta g_{1}\right)\left(\frac{1}{\mathbf{a}^{2}}\left|\frac{\partial m^{\eta}}{\partial u}\right|^{2}+\frac{1}{\mathbf{b}^{2}}\left|\frac{\partial m^{\eta}}{\partial v}\right|^{2}\right) d \mathbf{s} d u d v
\end{aligned}
$$


In particular, $\mathbf{m}_{0}^{\eta}$ does not depend on the transverse variables, so there exists a constant $C_{1}$ such that for all $\eta>0$,

$$
\mathcal{E}_{\eta}\left(m_{0}^{\eta}\right) \leq C_{1} \eta^{2}
$$

By properties of the applied field $\mathbf{h}_{a, \eta}$, we have for all $t$ :

$$
\left\|\mathbf{h}_{a, \eta}(t)\right\|_{L^{2}\left(\Omega_{\eta}\right)}^{2}=\eta^{2} \int_{[0, L]} \frac{\pi \mathbf{a b}}{1+\eta g_{1}}\left|h_{a}(t, \mathbf{s})\right|^{2} d \mathbf{s} .
$$

So, using (2.6), there exists $C_{2}$ such that for all $\eta$,

$$
\mathcal{G}_{\eta}(t) \leq C_{2} \eta^{2}\left(1+\int_{0}^{t}\left\|h_{a}(\tau, \cdot)\right\|_{L^{2}([0, L])}^{2} d \tau\right) .
$$

In addition, the current induced velocity $\overrightarrow{\mathbf{v}}_{\eta}(t)$ is uniformly bounded by $\|j(t, \cdot)\|_{L^{\infty}([0, L])}$. So there exists a constant $C_{3}$ such that for all $t$,

$$
\mathcal{V}_{\eta}(t) \leq C_{3} \int_{0}^{t}\|j(\tau, \cdot)\|_{L^{\infty}([0, L])}^{2} d \tau:=\nu(t)
$$

We define $G$ as:

$$
G(t)=\left(C_{1}+C_{2} \int_{0}^{t}\left\|h_{a}(\tau, \cdot)\right\|_{L^{2}([0, L])} d \tau\right)(1+\nu(t) \exp \nu(t)) .
$$

Therefore, using (2.7) and (2.8), we obtain by the energy formula that for all $t>0$ and for all $\eta>0$,

$$
\frac{\ell^{2}}{2}\left\|\nabla \mathbf{m}^{\eta}(t, \cdot)\right\|_{L^{2}\left(\Omega_{\eta}\right)}^{2}+\frac{\alpha}{2} \int_{0}^{t}\left\|\frac{\partial \mathbf{m}^{\eta}}{\partial t}(\tau, \cdot)\right\|_{L^{2}\left(\Omega_{\eta}\right)}^{2} d \tau \leq \eta^{2} G(t) .
$$

Also, with (2.5) we get that for all $T$ there exists a constant $C(T)$ such that for all $\eta>0$,

$$
\begin{array}{cc}
\left\|\frac{\partial m^{\eta}}{\partial u}\right\|_{L^{\infty}\left(0, T ; L^{2}(\mathcal{O})\right)}^{2}+\left\|\frac{\partial m^{\eta}}{\partial v}\right\|_{L^{\infty}\left(0, T ; L^{2}(\mathcal{O})\right)}^{2} & \leq C(T) \eta^{2}, \\
\left\|\frac{\partial m^{\eta}}{\partial \mathbf{s}}-g_{2} \frac{\partial m^{\eta}}{\partial u}-g_{3} \frac{\partial m^{\eta}}{\partial v}\right\|_{L^{\infty}\left(0, T ; L^{2}(\mathcal{O})\right)}^{2}+\left\|\frac{\partial m^{\eta}}{\partial t}\right\|_{L^{2}([0, T] \times \mathcal{O})}^{2} & \leq C(T) .
\end{array}
$$

Concerning the demagnetizing field, for a given $w: \mathcal{O} \longrightarrow \mathbb{R}^{3}$, we define $\mathbf{w}: \Omega_{\eta} \longrightarrow \mathbb{R}^{3}$ by $\mathbf{w} \circ \Psi_{\eta}=w$, and we define $h_{\eta}(w): \mathcal{O} \longrightarrow \mathbb{R}^{3}$ by:

$$
\mathbf{H}_{d}(\mathbf{w}) \circ \Psi_{\eta}=h_{\eta}(w) .
$$

Since $-\mathbf{H}_{d}$ is an orthogonal projection for the $L^{2}\left(\mathbb{R}^{3}\right)$ inner product, then we have for all $w \in L^{2}(\mathcal{O})$ :

$$
\left\|\mathbf{H}_{d}(\mathbf{w})\right\|_{L^{2}\left(\Omega_{\eta}\right)}^{2} \leq\left\|\mathbf{H}_{d}(\mathbf{w})\right\|_{L^{2}\left(\mathbb{R}^{3}\right)}^{2} \leq\|\mathbf{w}\|_{L^{2}\left(\Omega_{\eta}\right)}^{2}
$$

so in the rescaled variables, we obtain that there exists a constant $K$ such that for all $w$ and all $\eta>0$ :

$$
\left\|h_{\eta}(w)\right\|_{L^{2}(\mathcal{O})}^{2} \leq K\|w\|_{L^{2}(\mathcal{O})}^{2} .
$$

In addition, if $w \in L^{\infty}(\mathcal{O})$ takes its values in the unit sphere, then there exists a constant $K^{\prime}$ independent of $w$ such that for all $\eta$,

$$
\left\|h_{\eta}(w)\right\|_{L^{2}(\mathcal{O})}^{2} \leq K^{\prime} .
$$

In particular, denoting by $H^{\eta}(t, \cdot)=h_{\eta}\left(m^{\eta}(t, \cdot)\right)$, from the previous estimates, we obtain that for all $\eta>0$,

$$
\left\|H^{\eta}\right\|_{L^{\infty}\left(\mathbb{R}^{+} ; L^{2}(\mathcal{O})\right)} \leq K^{\prime} .
$$

Therefore, using Estimates (2.9) and (2.14), there exists a subsequence that we also denote by $m^{\eta}$ such that for all $T$, 
1. $m^{\eta} \rightarrow m$ in $L^{\infty}\left(0, T ; H^{1}(\mathcal{O})\right)$ weak *,

2. $\frac{\partial m^{\eta}}{\partial u} \longrightarrow 0$ and $\frac{\partial m^{\eta}}{\partial u} \longrightarrow 0$ in $\left.L^{\infty}\left(0, T ; L^{2}(\mathcal{O})\right)\right)$,

3. $\frac{\partial m^{\eta}}{\partial t} \rightarrow \frac{\partial m}{\partial t}$ weak in $L^{2}([0, T] \times \mathcal{O})$,

4. $H^{\eta} \rightarrow H$ in $L^{\infty}\left(0, T ; L^{2}(\mathcal{O})\right)$ weak *.

By using the Aubin Simon Lemma, we conclude that $m^{\eta}$ strongly tends to $m$ in $L^{\infty}\left(0, T ; L^{p}(\mathcal{O})\right)$ for $p \in\left[2,6\left[\right.\right.$ and by extracting a subsequence, we can assume that $m^{\eta} \longrightarrow m$ almost everywhere. In particular, we obtain that $|m|=1$ a.e. on $\mathbb{R}^{+} \times \mathcal{O}$.

\section{Limit in the weak formulation}

In this section, we aim to obtain the limit in the weak formulation by rewriting the formulation (1.8) in the new variables $(\mathbf{s}, u, v)$ and taking the limit when $\eta$ tends to zero. On the one hand, since the current $\overrightarrow{\mathbf{v}}_{\eta}$ is given by (1.10), we have

$$
\left(\left(\mathbf{v}_{\eta} \cdot \nabla\right) \mathbf{m}^{\eta}\right)\left(\Psi_{\eta}(\mathbf{s}, u, v)\right)=j(t, \mathbf{s}) d \mathbf{m}^{\eta}\left(\Psi_{\eta}(\mathbf{s}, u, v)\right)\left(\Gamma^{\prime}(\mathbf{s})\right) .
$$

On the other hand, we remark that for all orthonormal frame $\left(\vec{\xi}_{1}, \vec{\xi}_{2}, \vec{\xi}_{3}\right)$, we have:

$$
\sum_{i=1}^{3}\left(\mathbf{m}^{\eta}(\mathbf{x}) \times \frac{\partial \mathbf{m}^{\eta}}{\partial x_{i}}(\mathbf{x})\right) \cdot \frac{\partial \Phi}{\partial x_{i}}(\mathbf{x})=\sum_{i=1}^{3}\left(\mathbf{m}^{\eta}(\mathbf{x}) \times d \mathbf{m}^{\eta}(\mathbf{x})\left(\vec{\xi}_{i}\right)\right) \cdot d \Phi(\mathbf{x})\left(\vec{\xi}_{i}\right) .
$$

We take $\vec{\xi}_{1}=\Gamma^{\prime}(\mathbf{s}), \vec{\xi}_{2}=\overrightarrow{\mathbf{e}}_{\mathbf{a}}(\mathbf{s}), \vec{\xi}_{3}=\overrightarrow{\mathbf{e}}_{\mathbf{b}}(\mathbf{s})$, and $\Phi$ of the form $\Phi\left(t, \Psi_{\eta}(\mathbf{s}, u, v)\right)=\phi(t, \mathbf{s})$.

Since $\phi$ depends only on $\mathbf{s}$, we have:

$$
\begin{aligned}
& \frac{\partial \phi}{\partial u}=d \Phi\left(t, \Psi_{\eta}(\mathbf{s}, u, v)\right)\left(\frac{\partial \Psi_{\eta}}{\partial u}(\mathbf{s}, u, v)\right)=\eta \mathbf{a} d \Phi\left(t, \Psi_{\eta}(\mathbf{s}, u, v)\right)\left(\overrightarrow{\mathbf{e}_{\mathbf{a}}}\right)=0 \\
& \frac{\partial \phi}{\partial v}=d \Phi\left(t, \Psi_{\eta}(\mathbf{s}, u, v)\right)\left(\frac{\partial \Psi_{\eta}}{\partial v}(\mathbf{s}, u, v)\right)=\eta \mathbf{b} d \Phi\left(t, \Psi_{\eta}(\mathbf{s}, u, v)\right)\left(\overrightarrow{\mathbf{e}_{\mathbf{b}}}\right)=0 .
\end{aligned}
$$

Thus, we obtain:

$$
d \Phi\left(t, \Psi_{\eta}(\mathbf{s}, u, v)\right)\left(\overrightarrow{\mathbf{e}_{\mathbf{a}}}\right)=d \Phi\left(t, \Psi_{\eta}(\mathbf{s}, u, v)\right)\left(\overrightarrow{\mathbf{e}_{\mathbf{b}}}\right)=0 .
$$

Next, differentiating $\phi$ with respect to $\mathbf{s}$ and by (2.1) and (3.1), we obtain:

$$
\frac{\partial \phi}{\partial \mathbf{s}}(t, \mathbf{s})=d \Phi\left(t, \Psi_{\eta}(\mathbf{s}, u, v)\left(\frac{\Psi_{\eta}}{\partial \mathbf{s}}\right)=\left(1+\eta g_{1}\right) d \Phi\left(t, \Psi_{\eta}(\mathbf{s}, u, v)\right)\left(\Gamma^{\prime}(\mathbf{s})\right) .\right.
$$

Hence, we conclude that:

$$
\begin{aligned}
\sum_{i=1}^{3}\left(\mathbf{m}^{\eta}(\mathbf{x}) \times d \mathbf{m}^{\eta}(\mathbf{x})\left(\vec{\xi}_{i}\right)\right) \cdot d \Phi(\mathbf{x})\left(\vec{\xi}_{i}\right) & =\left(\mathbf{m}^{\eta}(\mathbf{x}) \times d \mathbf{m}^{\eta}(\mathbf{x})\left(\Gamma^{\prime}(\mathbf{s})\right)\right) \cdot d \Phi(\mathbf{x})\left(\Gamma^{\prime}(\mathbf{s})\right) \\
& =\frac{1}{1+\eta g_{1}}\left(m^{\eta}(\mathbf{x}) \times\left(\frac{\partial m^{\eta}}{\partial \mathbf{s}}-\frac{g_{2}}{\mathbf{a}} \frac{\partial m^{\eta}}{\partial u}-\frac{g_{2}}{\mathbf{b}} \frac{\partial m^{\eta}}{\partial u}\right)\right) \frac{\partial \phi}{\partial \mathbf{s}}
\end{aligned}
$$

Then, we can write the weak formulation as follows: 


$$
\begin{aligned}
\int_{\mathbb{R}^{+} \times \mathcal{O}} \mathbf{a}(\mathbf{s}) \mathbf{b}(\mathbf{s}) & \left(1+\eta g_{1}(\mathbf{s}, u, v)\right)\left(\frac{\partial m^{\eta}}{\partial t}-\alpha m^{\eta} \times \frac{\partial m^{\eta}}{\partial t}\right) \cdot \phi(t, \mathbf{s})= \\
& \int_{\mathbb{R}^{+} \times \mathcal{O}} \ell^{2} \mathbf{a}(\mathbf{s}) \mathbf{b}(\mathbf{s})\left(m^{\eta} \times\left(\frac{\partial m^{\eta}}{\partial \mathbf{s}}-\frac{g_{2}}{\mathbf{a}} \frac{\partial m^{\eta}}{\partial u}-\frac{g_{3}}{\mathbf{b}} \frac{\partial m^{\eta}}{\partial v}\right)\right) \frac{\partial \phi}{\partial \mathbf{s}} \\
& -\int_{\mathbb{R}^{+} \times \mathcal{O}} \mathbf{a}(\mathbf{s}) \mathbf{b}(\mathbf{s})\left(1+\eta g_{1}(\mathbf{s}, u, v)\right) m^{\eta} \times\left(H^{\eta}+h_{a}\right) \cdot \phi(t, \mathbf{s}) \\
- & \int_{\mathbb{R}^{+} \times \mathcal{O}} \mathbf{a}(\mathbf{s}) \mathbf{b}(\mathbf{s}) j(t, \mathbf{s})\left(\frac{\partial m^{\eta}}{\partial \mathbf{s}}-\frac{g_{2}}{\mathbf{a}} \frac{\partial m^{\eta}}{\partial u}-\frac{g_{3}}{\mathbf{b}} \frac{\partial m^{\eta}}{\partial v}\right) \cdot \phi(t, \mathbf{s})
\end{aligned}
$$

Taking the limit when $\eta$ tends to zero and using that

- $m^{\eta}$ tends to $m$ strongly in $L^{\infty}\left(0, T ; L^{2}(\mathcal{O})\right)$,

- $\frac{\partial m^{\eta}}{\partial \mathbf{s}}$ and $H^{\eta}$ tend respectively to $\frac{\partial m}{\partial \mathbf{s}}$ and $H$ in $L^{\infty}\left(0, T ; L^{2}(\mathcal{O})\right)$ weak *,

- $\frac{\partial m^{\eta}}{\partial t}$ tends weakly to $\frac{\partial m}{\partial t}$ in $L^{2}\left(0, T ; L^{2}(\mathcal{O})\right)$,

- $\frac{\partial m^{\eta}}{\partial u}$ and $\frac{\partial m^{\eta}}{\partial v}$ tend to zero strongly in $L^{\infty}\left(0, T ; L^{2}(\mathcal{O})\right)$,

- $m$ does not depend on the transverse variables $u$ and $v$,

we obtain the following formulation:

$$
\begin{array}{r}
\int_{\mathbb{R}^{+\times[0, L]}} \sigma\left(\frac{\partial m}{\partial t}-\alpha m \times \frac{\partial m}{\partial t}\right) \cdot \phi d t d \mathbf{s}=\int_{\mathbb{R}^{+} \times[0, L]} \ell^{2} \sigma\left(m \times \frac{\partial m}{\partial \mathbf{s}}\right) \cdot \frac{\partial \phi}{\partial \mathbf{s}} d t d \mathbf{s} \\
-\int_{\mathbb{R}^{+} \times[0, L]} \sigma m \times\left(H+h_{a}\right) \cdot \phi d t d \mathbf{s}-\int_{\mathbb{R}^{+} \times[0, L]} \sigma j \frac{\partial m}{\partial \mathbf{s}} \cdot \phi d t d \mathbf{s} .
\end{array}
$$

where $\sigma(\mathbf{s})=\pi \mathbf{a}(\mathbf{s}) \mathbf{b}(\mathbf{s})$ is the rescaled area of the section. It remains to characterize the limit $H$ of the rescaled demagnetizing field.

\section{Limit for the Demagnetizing Field}

Recall that we denoted by $H^{\eta}$ the demagnetizing field induced by $\mathbf{m}^{\eta}$ written in the variables $(\mathbf{s}, U) \in[0, L] \times B_{2}(0,1): H^{\eta}(t, \mathbf{s}, U)=\mathbf{H}_{d}\left(\mathbf{m}^{\eta}(t, \cdot)\right)\left(\Psi^{\eta}(\mathbf{s}, U)\right)$. We introduce $H_{\eta}=h_{\eta}(m(t, \cdot))$. From (2.12), for all $\eta>0$ :

$$
\left\|H^{\eta}-H_{\eta}\right\|_{L^{2}(\mathcal{O})} \leq K\left\|m^{\eta}-m\right\|_{L^{2}(\mathcal{O})} .
$$

So in order to describe the limit of $H^{\eta}$, we will study the limit of $H_{\eta}$ when $\eta$ tends to zero. We claim the following Proposition.

Proposition 4.1. Let $w \in \mathcal{C}^{1}\left(\overline{\mathcal{O}} ; \mathbb{R}^{3}\right)$. We assume that $w$ is independent of the $(u, v)$ variables, so that $w(\mathbf{s}, u, v)=w_{1}(\mathbf{s}) \Gamma^{\prime}(\mathbf{s})+w_{2}(\mathbf{s}) \overrightarrow{\mathbf{e}}_{\mathbf{a}}(\mathbf{s})+w_{3}(\mathbf{s}) \overrightarrow{\mathbf{e}}_{\mathbf{b}}(\mathbf{s})$. Then when $\eta$ tends to zero, $h_{\eta}(w)$ tends strongly in $L^{2}(\mathcal{O})$ to $H_{d}(w)$ given by

$$
H_{d}(w)=-\frac{\mathbf{b}(\mathbf{s})}{\mathbf{a}(\mathbf{s})+\mathbf{b}(\mathbf{s})}\left(w \cdot \overrightarrow{\mathbf{e}}_{\mathbf{a}}\right) \overrightarrow{\mathbf{e}}_{\mathbf{a}}-\frac{\mathbf{a}(\mathbf{s})}{\mathbf{a}(\mathbf{s})+\mathbf{b}(\mathbf{s})}\left(w \cdot \overrightarrow{\mathbf{e}}_{\mathbf{b}}\right) \overrightarrow{\mathbf{e}}_{\mathbf{b}}
$$


Since $m$ does not depend on the transverse variables, using the density of $\mathcal{C}^{1}([0, L])$ in $L^{2}([0, L])$, and (2.12), we obtain through Proposition 4.1 that $H_{\eta}$ tends to $H_{d}(m)$ strongly in $L^{2}(\Omega)$, and we conclude the proof of Theorem 1.1. So it remains to establish Proposition 4.1.

Proof of Proposition 4.1: we define $\mathbf{w}_{\eta}: \Omega_{\eta} \longrightarrow \mathbb{R}^{3}$ by $\mathbf{w}_{\eta} \circ \Psi_{\eta}=w$. Recall that $h_{\eta}(w)$ is given by $h_{\eta}(w)=\mathbf{H}_{d}\left(\mathbf{w}_{\eta}\right) \circ \Psi_{\eta}$. Since $\operatorname{curl} \mathbf{H}_{d}\left(\mathbf{w}_{\eta}\right)=0$, we can write $\mathbf{H}_{d}\left(\mathbf{w}_{\eta}\right)=-\nabla \phi$ with $\Delta \phi=\operatorname{div} \overline{\mathbf{w}_{\eta}}$, where $\overline{\mathbf{w}_{\eta}}$ is the extension of $\mathbf{w}_{\eta}$ by zero outside $\Omega_{\eta}$. From the last equation, we have $\phi=-G * \operatorname{div} \overline{\mathbf{w}_{\eta}}$ where $G(x)=\frac{1}{4 \pi|x|} \in L_{\text {loc }}^{1}\left(\mathbb{R}^{3}\right)$ is the fundamental solution for the operator $-\Delta$ in $\mathbb{R}^{3}$. Therefore we have:

$$
\mathbf{H}_{d}\left(\mathbf{w}_{\eta}\right)(\mathbf{x})=-\frac{1}{4 \pi} \int_{\Omega_{\eta}} \frac{\mathbf{x}-\mathbf{y}}{|\mathbf{x}-\mathbf{y}|^{3}} \operatorname{div} \mathbf{w}_{\eta}(\mathbf{y}) d \mathbf{y}+\frac{1}{4 \pi} \int_{\partial \Omega_{\eta}} \frac{\mathbf{x}-\mathbf{y}}{|\mathbf{x}-\mathbf{y}|^{3}}\left\langle\mathbf{w}_{\eta}(\mathbf{y}) \mid \nu(\mathbf{y})\right\rangle d \sigma(\mathbf{y}),
$$

where $\nu(\mathbf{y})$ is the outward unit normal on $\partial \Omega_{\eta}$.

Writting $w(\mathbf{s}, u, v)=w_{1}(\mathbf{s}) \Gamma^{\prime}(\mathbf{s})+w_{2}(\mathbf{s}) \overrightarrow{\mathbf{e}}_{\mathbf{a}}(\mathbf{s})+w_{3}(\mathbf{s}) \overrightarrow{\mathbf{e}}_{\mathbf{b}}(\mathbf{s})$, then, by $(2.3)$ we have:

$$
\begin{aligned}
w(\mathbf{s}, u, v)= & w_{1}\left(\frac{1}{1+\eta g_{1}}\left(\frac{\partial \Psi_{\eta}}{\partial \mathbf{s}}-\frac{g_{2}}{\mathbf{a}} \frac{\partial \Psi_{\eta}}{\partial u}-\frac{g_{3}}{\mathbf{b}} \frac{\partial \Psi_{\eta}}{\partial v}\right)\right)+w_{2}\left(\frac{1}{\eta \mathbf{a}} \frac{\partial \Psi_{\eta}}{\partial u}\right)+w_{3}\left(\frac{1}{\eta \mathbf{b}} \frac{\partial \Psi_{\eta}}{\partial v}\right) \\
= & : \frac{\partial \Psi_{\eta}}{\partial \mathbf{s}} \gamma_{1}+\frac{\partial \Psi_{\eta}}{\partial u} \gamma_{2}+\frac{\partial \Psi_{\eta}}{\partial v} \gamma_{3} .
\end{aligned}
$$

Now, we compute Gram's matrix given by:

$$
G(\mathbf{s}, u, v)=\left(\begin{array}{ccc}
\left\langle\frac{\partial \Psi_{\eta}}{\partial \mathbf{s}} \mid \frac{\partial \Psi_{\eta}}{\partial \mathbf{s}}\right\rangle & \left\langle\frac{\partial \Psi_{\eta}}{\partial \mathbf{s}} \mid \frac{\partial \Psi_{\eta}}{\partial u}\right\rangle & \left\langle\frac{\partial \Psi_{\eta}}{\partial \mathbf{s}} \mid \frac{\partial \Psi_{\eta}}{\partial v}\right\rangle \\
\left\langle\frac{\partial \Psi_{\eta}}{\partial \mathbf{s}} \mid \frac{\partial \Psi_{\eta}}{\partial u}\right\rangle & \left\langle\frac{\partial \Psi_{\eta}}{\partial u} \mid \frac{\partial \Psi_{\eta}}{\partial u}\right\rangle & \left\langle\frac{\partial \Psi_{\eta}}{\partial u} \mid \frac{\partial \Psi_{\eta}}{\partial v}\right\rangle \\
\left\langle\frac{\partial \Psi_{\eta}}{\partial \mathbf{s}} \mid \frac{\partial \Psi_{\eta}}{\partial v}\right\rangle & \left\langle\frac{\partial \Psi_{\eta}}{\partial u} \mid \frac{\partial \Psi_{\eta}}{\partial v}\right\rangle & \left\langle\frac{\partial \Psi_{\eta}}{\partial v} \mid \frac{\partial \Psi_{\eta}}{\partial v}\right\rangle
\end{array}\right)=\left(\begin{array}{ccc}
\left(1+\eta g_{1}\right)^{2}+\eta^{2}\left(g_{2}+g_{3}\right)^{2} & \eta^{2} \mathbf{a} g_{2} & \eta^{2} \mathbf{b} g_{3} \\
\eta^{2} \mathbf{a} g_{2} & \eta^{2} \mathbf{a}^{2} & 0 \\
\eta^{2} \mathbf{b} g_{3} & 0 & \eta^{2} \mathbf{b}^{2}
\end{array}\right),
$$

so that $\operatorname{det} \mathrm{G}=\eta^{4} \mathbf{a}^{2} \mathbf{b}^{2}\left(1+\eta g_{1}\right)^{2}$.

Since $w(\mathbf{s}, u, v)=\frac{\partial \Psi_{\eta}}{\partial \mathbf{s}} \gamma_{1}+\frac{\partial \Psi_{\eta}}{\partial u} \gamma_{2}+\frac{\partial \Psi_{\eta}}{\partial v} \gamma_{3}$, then

$$
\operatorname{div} \mathbf{w}_{\eta}\left(\Psi_{\eta}(s, u, v)\right)=\frac{1}{\sqrt{\operatorname{det} G}}\left(\frac{\partial}{\partial \mathbf{s}}\left(\gamma_{1} \sqrt{\operatorname{det} G}\right)+\frac{\partial}{\partial u}\left(\gamma_{2} \sqrt{\operatorname{det} G}\right)+\frac{\partial}{\partial v}\left(\gamma_{3} \sqrt{\operatorname{det} G}\right)\right) .
$$

By direct substitution, we can see that

$$
\begin{aligned}
\operatorname{div} \mathbf{w}_{\eta}\left(\Psi_{\eta}(s, u, v)\right) & =\frac{1}{\eta^{2} \mathbf{a b}\left(1+g_{1}\right)}\left[\frac{\partial}{\partial \mathbf{s}}\left(\frac{w_{1}}{1+\eta g_{1}} \eta^{2} \mathbf{a b}\left(1+\eta g_{1}\right)\right)\right. \\
& +\frac{\partial}{\partial u}\left(\left(\frac{w_{2}}{\eta \mathbf{a}}-\frac{g_{2} w_{1}}{\mathbf{a}\left(1+g_{1}\right)}\right) \eta^{2} \mathbf{a b}\left(1+\eta g_{1}\right)\right) \\
& \left.+\frac{\partial}{\partial v}\left(\left(\frac{w_{2}}{\eta \mathbf{a}}-\frac{g_{2} w_{1}}{\mathbf{a}\left(1+g_{1}\right)}\right) \eta^{2} \mathbf{a b}\left(1+\eta g_{1}\right)\right)\right] \\
& =\frac{1}{\mathbf{a b}\left(1+\eta g_{1}\right)}\left(\frac{\partial\left(w_{1} \mathbf{a b}\right)}{\partial \mathbf{s}}-w_{1}\left(\mathbf{b a}^{\prime}+\mathbf{a b}^{\prime}\right)-r_{1} \mathbf{a b} w_{2}-r_{2} \mathbf{a b} w_{3}\right) .
\end{aligned}
$$

So, we obtain that:

$$
\operatorname{div} \mathbf{w}_{\eta}\left(\Psi_{\eta}(\mathbf{s}, U)\right)=\frac{1}{1+\eta g_{1}(\mathbf{s}, U)}\left(\frac{\partial w_{1}}{\partial \mathbf{s}}(\mathbf{s})-r_{1}(\mathbf{s}) w_{2}(\mathbf{s})-r_{2}(\mathbf{s}) w_{3}(\mathbf{s})\right) .
$$

We split $\partial \Omega_{\eta}$ as $\Psi_{\eta}\left(\{0\} \times B_{2}(0,1)\right) \cup \Psi_{\eta}\left(\{L\} \times B_{2}(0,1)\right) \cup \Psi_{\eta}\left([0, L] \times \partial B_{2}(0,1)\right):=\Gamma_{1} \cup \Gamma_{2} \cup \Gamma_{3}$. So, we can rewrite (4.1) in the variables $(\mathbf{s}, u, v)$ as follows:

$$
H_{d}\left(\mathbf{w}_{\eta}\right)(\Psi(\mathbf{s}, U))=H_{\eta}(\mathbf{s}, U)=I_{1}^{\eta}(\mathbf{s}, U)+I_{2}^{\eta}(\mathbf{s}, U)+I_{3}^{\eta}(\mathbf{s}, U)+I_{4}^{\eta}(\mathbf{s}, U),
$$

where 
- $I_{1}^{\eta}=-\frac{\eta^{2}}{4 \pi} \int_{\mathcal{O}} \frac{\Psi_{\eta}(\mathbf{s}, U)-\Psi_{\eta}\left(\mathbf{s}^{\prime}, U^{\prime}\right)}{\left|\Psi_{\eta}(\mathbf{s}, U)-\Psi_{\eta}\left(\mathbf{s}^{\prime}, U^{\prime}\right)\right|^{3}}\left(\frac{\partial w_{1}}{\partial \mathbf{s}}\left(\mathbf{s}^{\prime}\right)-r_{1}\left(\mathbf{s}^{\prime}\right) w_{2}\left(\mathbf{s}^{\prime}\right)-r_{2}\left(\mathbf{s}^{\prime}\right) w_{3}\left(\mathbf{s}^{\prime}\right)\right) \sigma\left(\mathbf{s}^{\prime}\right) d \mathbf{s}^{\prime} d U^{\prime}$

- $I_{2}^{\eta}=-\frac{\eta^{2}}{4 \pi} \int_{B_{2}(0,1)} \frac{\Psi_{\eta}(s, U)-\Psi_{\eta}\left(0, U^{\prime}\right)}{\left|\Psi_{\eta}(s, U)-\Psi_{\eta}\left(0, U^{\prime}\right)\right|^{3}} \sigma(0) w_{1}(0) d U^{\prime}$

- $I_{3}^{\eta}=\frac{\eta^{2}}{4 \pi} \int_{B_{2}(0,1)} \frac{\Psi_{\eta}(s, U)-\Psi_{\eta}\left(L, U^{\prime}\right)}{\left|\Psi_{\eta}(s, U)-\Psi_{\eta}\left(L, U^{\prime}\right)\right|^{3}} \sigma(L) w_{1}(L) d U^{\prime}$,

- $I_{4}^{\eta}=\frac{\eta}{4 \pi} \int_{0}^{L} \int_{0}^{2 \pi} \frac{\Psi_{\eta}(\mathbf{s}, U)-\Psi_{\eta}\left(\mathbf{s}^{\prime}, \overrightarrow{\mathbf{e}}_{r}(\theta)\right)}{\left|\Psi_{\eta}(\mathbf{s}, U)-\Psi_{\eta}\left(\mathbf{s}^{\prime}, \overrightarrow{\mathbf{e}}_{r}(\theta)\right)\right|^{3}} A\left(\mathbf{s}^{\prime}, \theta\right) G_{\eta}\left(\mathbf{s}^{\prime}, \theta\right) d \mathbf{s}^{\prime} d \theta$

with

$$
\overrightarrow{\mathbf{e}}_{r}(\theta)=(\cos \theta, \sin \theta), \quad A\left(\mathbf{s}^{\prime}, \theta\right)=\frac{\mathbf{b}\left(\mathbf{s}^{\prime}\right) w_{2}\left(\mathbf{s}^{\prime}\right) \cos \theta+\mathbf{a}\left(\mathbf{s}^{\prime}\right) w_{3}\left(\mathbf{s}^{\prime}\right) \sin \theta}{\left(\mathbf{b}^{2}\left(\mathbf{s}^{\prime}\right) \cos ^{2} \theta+\mathbf{a}^{2}\left(\mathbf{s}^{\prime}\right) \sin ^{2} \theta\right)^{\frac{1}{2}}},
$$

and $G_{\eta}\left(\mathbf{s}^{\prime}, \theta\right)=\left(\alpha_{1}\left(\mathbf{s}^{\prime}, \theta\right) \alpha_{2}\left(\mathbf{s}^{\prime}, \theta\right)-\left(\alpha_{3}\left(\mathbf{s}^{\prime}, \theta\right)\right)^{2}\right)^{\frac{1}{2}}$.

Lemma 4.1. There exists $C>0$ and $\eta_{0}>0$ such that for all $\eta \leq \eta_{0}, \mathbf{s}, \mathbf{s}^{\prime} \in[0, L]$ and $U, U^{\prime} \in$ $B_{2}(0,1)$ we have

$$
\left\|\Psi_{\eta}(\mathbf{s}, U)-\Psi_{\eta}\left(\mathbf{s}^{\prime}, U^{\prime}\right)\right\|_{\mathbb{R}^{3}} \geq C\left(\left|\mathbf{s}-\mathbf{s}^{\prime}\right|^{2}+\eta^{2}\left\|U-U^{\prime}\right\|^{2}\right)^{\frac{1}{2}} .
$$

Proof of Lemma 4.1. Assume that this Lemma is false. Then for all $n \geq 0$, there exists $\eta_{n} \leq \frac{1}{n}$, $\mathbf{s}_{n}, \mathbf{s}_{n}^{\prime} \in[0, L]$ and $U_{n}, U_{n}^{\prime} \in B_{2}(0,1)$ such that

$$
\left\|\Psi_{\eta_{n}}\left(\mathbf{s}_{n}, U_{n}\right)-\Psi_{h}\left(\mathbf{s}_{n}^{\prime}, U_{n}^{\prime}\right)\right\|<\frac{1}{n}\left(\left|\mathbf{s}_{n}-\mathbf{s}_{n}^{\prime}\right|^{2}+\left(\eta_{n}\right)^{2}\left\|U_{n}-U_{n}^{\prime}\right\|^{2}\right)^{\frac{1}{2}},
$$

which implies that $\left\|X\left(\mathbf{s}_{n}\right)-X\left(\mathbf{s}_{n}^{\prime}\right)\right\|$ tends to zero. By extracting a subsequence we can assume that $\mathbf{s}_{n}$ and $\mathbf{s}_{n}^{\prime}$ tend respectively to $\mathbf{s}_{\infty}$ and $\mathbf{s}_{\infty}^{\prime}$. Since $\left\|X\left(\mathbf{s}_{n}\right)-X\left(\mathbf{s}_{n}^{\prime}\right)\right\| \longrightarrow 0$, then $X\left(\mathbf{s}_{\infty}\right)=X\left(\mathbf{s}_{\infty}^{\prime}\right)$, which implies that $s_{\infty}=s_{\infty}^{\prime}$. Therefore $\left(\mathbf{s}_{n}, \eta_{n} U_{n}\right)_{n},\left(\mathbf{s}_{n}^{\prime}, \eta_{n} U_{n}^{\prime}\right)_{n}$ tends to $\left(\mathbf{s}_{\infty}, 0\right)$.

Next we define $\Psi:(\mathbf{s}, U) \mapsto \Gamma(s)+u \mathbf{a}(\mathbf{s}) \overrightarrow{\mathbf{e}}_{\mathbf{a}}(\mathbf{s})+v \mathbf{b}(\mathbf{s}) \overrightarrow{\mathbf{e}}_{\mathbf{b}}(\mathbf{s})$. So, by the local inversion theorem, there exists $\nu>0$ such that $\Psi$ is a $\mathcal{C}^{1}$-diffeomorphism from $\left[\mathbf{s}_{\infty}-\nu, \mathbf{s}_{\infty}+\nu\right] \times B_{2}(0, \nu)$ into its range $\mathcal{V}$. Even if it means reducing $\nu$, we can assume that $\Psi^{-1}$ is Lipschitz on $\mathcal{V}$ :

$$
\exists C, \forall(\mathbf{x}, \mathbf{y}) \in \mathcal{V} \times \mathcal{V},\left\|\Psi^{-1}(\mathbf{x})-\Psi^{-1}(\mathbf{y})\right\| \leq C\|\mathbf{x}-\mathbf{y}\| .
$$

For $n$ large enough, $\left(\mathbf{s}_{n}, \eta_{n} U_{n}\right)$ and $\left(\mathbf{s}_{n}^{\prime}, \eta_{n} U_{n}^{\prime}\right)$ are in $\left[\mathbf{s}_{\infty}-\nu, \mathbf{s}_{\infty}+\nu\right] \times B_{2}(0, \nu)$, so by applying (4.3) for $\mathbf{x}=\Psi_{\eta_{n}}\left(\mathbf{s}_{n}, U_{n}\right)$ and $\mathbf{y}=\Psi_{\eta_{n}}\left(\mathbf{s}_{n}^{\prime}, U_{n}^{\prime}\right)$, we obtain that

$$
\left(\left|\mathbf{s}_{n}-\mathbf{s}_{n}^{\prime}\right|^{2}+\left(\eta_{n}\right)^{2}\left\|U_{n}-U_{n}^{\prime}\right\|^{2}\right)^{\frac{1}{2}} \leq C\left\|\Psi_{\eta_{n}}\left(\mathbf{s}_{n}, U_{n}\right)-\Psi_{h}\left(\mathbf{s}_{n}^{\prime}, U_{n}^{\prime}\right)\right\|
$$

which together with (4.2) implies that $1<C \frac{1}{n}$ for $n$ large enough, which is a contradiction. This concludes the proof of Lemma 4.1.

We first prove that $I_{2}^{\eta}$ and $I_{3}^{\eta}$ tend to zero in $L^{2}(\mathcal{O})$ when $\eta$ tends to zero. By Lemma 4.1,

$$
\begin{aligned}
\left|I_{2}^{\eta}(\mathbf{s}, U)\right| & \leq K \eta^{2} \int_{B_{2}(0,1)} \frac{1}{\mathbf{s}^{2}+\eta^{2}\left\|U-U^{\prime}\right\|^{2}} d U^{\prime} \leq K \eta^{2} \int_{B_{2}(0,2)} \frac{1}{\mathbf{s}^{2}+\eta^{2}\left\|U^{\prime}\right\|^{2}} d U^{\prime} \\
& \leq 2 \pi K \eta^{2} \int_{0}^{2} \frac{r}{\mathbf{s}^{2}+\eta^{2} r^{2}} d r \leq \pi K\left(\ln \left(\mathbf{s}^{2}+4 \eta^{2}\right)-\ln \mathbf{s}^{2}\right) .
\end{aligned}
$$

Clearly, the right hand side of the previous inequality strongly tends to zero in $L^{2}(\mathcal{O})$ when $\eta$ tends to zero, so $I_{2}^{\eta}$ tends to zero in $L^{2}(\mathcal{O})$. In the same way, we prove the same result for $I_{3}^{\eta}$. 
Since $w \in \mathcal{C}^{1}(\overline{\mathcal{O}})$, we can bound $I_{1}^{\eta}$ by the same arguments:

$\left|I_{1}^{\eta}(\mathbf{s}, U)\right| \leq K \eta^{2} \int_{0}^{L} \int_{B_{2}(0,1)} \frac{1}{\left(\mathbf{s}-\mathbf{s}^{\prime}\right)^{2}+\eta^{2}\left\|U-U^{\prime}\right\|^{2}} d U^{\prime} d \mathbf{s}^{\prime} \leq 2 \pi K \int_{0}^{L}\left(\ln \left(\left(\mathbf{s}^{\prime}\right)^{2}+4 \eta^{2}\right)-\ln \left(\mathbf{s}^{\prime}\right)^{2}\right) d \mathbf{s}^{\prime}$.

The right hand side of the previous estimate does not depend on $(\mathbf{s}, U)$ and tends to zero when $\eta$ tends to zero, so $I_{1}^{\eta}$ tends to zero uniformly on $\Omega$, and it strongly tends to zero in $L^{2}(\Omega)$.

Now, we split $I_{4}^{\eta}$ in 2 parts: $I_{4}^{\eta}=I_{4,1}^{\eta}+I_{4,2}^{\eta}$ with:

$$
\begin{aligned}
I_{4,1}^{\eta} & =\frac{\eta}{4 \pi} \int_{0}^{2 \pi} \int_{0}^{L} \frac{\Psi_{\eta}\left(\mathbf{s}, \overrightarrow{\mathbf{e}}_{r}(\theta)\right)-\Psi_{\eta}\left(\mathbf{s}^{\prime}, \overrightarrow{\mathbf{e}}_{r}(\theta)\right)}{\left|\Psi_{\eta}(\mathbf{s}, U)-\Psi_{\eta}\left(\mathbf{s}^{\prime}, \overrightarrow{\mathbf{e}}_{r}(\theta)\right)\right|^{3}} A\left(\mathbf{s}^{\prime}, \theta\right) G_{\eta}\left(\mathbf{s}^{\prime}, \theta\right) d \mathbf{s}^{\prime} d \theta, \\
I_{4,2}^{\eta} & =\frac{\eta^{2}}{4 \pi} \int_{0}^{2 \pi} \int_{0}^{L} \frac{\mathbf{a}(\mathbf{s}) \overrightarrow{\mathbf{e}}_{\mathbf{a}}(\mathbf{s})(u-\cos \theta)+\mathbf{b}(\mathbf{s}) \overrightarrow{\mathbf{e}}_{\mathbf{b}}(\mathbf{s})(v-\sin \theta)}{\left|\Psi_{\eta}(\mathbf{s}, U)-\Psi_{\eta}\left(\mathbf{s}^{\prime}, \overrightarrow{\mathbf{e}}_{r}(\theta)\right)\right|^{3}} A\left(\mathbf{s}^{\prime}, \theta\right) G_{\eta}\left(\mathbf{s}^{\prime}, \theta\right) d \mathbf{s}^{\prime} d \theta .
\end{aligned}
$$

By Lemma 4.1, we have:

$$
\begin{aligned}
\left|I_{4,1}^{\eta}\right| & \leq K \eta \int_{0}^{2 \pi} \int_{0}^{L} \frac{\left|\mathbf{s}-\mathbf{s}^{\prime}\right| d \theta d \mathbf{s}^{\prime}}{\left(\mathbf{s}-\mathbf{s}^{\prime}\right)^{2}+\eta^{2}\left\|U-\overrightarrow{\mathbf{e}}_{r}(\theta)\right\|^{2}} \leq 2 K \eta \int_{0}^{2 \pi} \int_{0}^{L} \frac{\mathbf{s}^{\prime} d \theta d \mathbf{s}^{\prime}}{\left(\mathbf{s}^{\prime}\right)^{2}+\eta^{2}\left\|U-\overrightarrow{\mathbf{e}}_{r}(\theta)\right\|^{2}} \\
& \left.\leq 4 \pi K \eta \int_{0}^{L} \frac{\mathbf{s}^{\prime}}{\left(\mathbf{s}^{\prime}\right)^{2}+\eta^{2}(\|U\|-1)^{2}} d \mathbf{s}^{\prime} d \theta \quad \leq 2 \pi \eta\left(\ln \left(L+\eta^{2}(\|U\|-1)^{2}\right)-\ln \eta^{2}(\|U\|-1)^{2}\right)\right) \\
& \leq 2 \pi \eta \ln (L+2)-4 \pi \eta \ln \eta-4 \pi \eta \ln (1-\|U\|) .
\end{aligned}
$$

When $\eta$ tends to zero, the right hand side term tends to zero in $L^{2}(\mathcal{O})$ so $I_{4,1}^{\eta}$ tends to zero strongly in $L^{2}(\mathcal{O})$.

For the last term, by Taylor expansion, we write $\Gamma\left(\mathbf{s}^{\prime}\right)-\Gamma(\mathbf{s})=\left(\mathbf{s}^{\prime}-\mathbf{s}\right) \Lambda\left(\mathbf{s}, \mathbf{s}^{\prime}\right)$ where $\Lambda\left(\mathbf{s}, \mathbf{s}^{\prime}\right)=$ $\int_{0}^{1} \Gamma^{\prime}\left(\mathbf{s}+\tau\left(\mathbf{s}^{\prime}-\mathbf{s}\right)\right) d \tau$ so that $\Lambda \in \mathcal{C}^{1}\left([0, L]^{2} ; \mathbb{R}^{3}\right)$. In addition, we denote by $\chi(\mathbf{s})$ the $3 \times 2$ matrix such that $\chi(\mathbf{s})(u, v)=\mathbf{a}(\mathbf{s}) \overrightarrow{\mathbf{e}}_{\mathbf{a}}(\mathbf{s}) u+\mathbf{b}(\mathbf{s}) \overrightarrow{\mathbf{e}}_{\mathbf{b}}(\mathbf{s}) v$.

Using change of variable $\mathbf{s}^{\prime}=\mathbf{s}+\tau \eta\left\|U-\overrightarrow{\mathbf{e}}_{r}(\theta)\right\|$ in the integral in $\mathbf{s}^{\prime}$, we obtain:

$$
I_{4,2}^{\eta}(\mathbf{s}, U)=\frac{1}{4 \pi} \int_{0}^{2 \pi} \int_{\frac{-\mathbf{s}}{\eta\left\|U-\mathbf{e}_{r}(\theta)\right\|}}^{\frac{L-s}{\eta \| U-\mathbf{e}_{r}(\theta) \Pi}} K_{\eta}(\mathbf{s}, U, \theta, \tau) d \tau d \theta,
$$

where

$$
\begin{aligned}
K_{\eta}(\mathbf{s}, U, \theta, \tau) & =\frac{\eta^{3}\left|U-\overrightarrow{\mathbf{e}}_{r}(\theta)\right| \chi(\mathbf{s})\left(U-\overrightarrow{\mathbf{e}}_{r}(\theta)\right) A\left(\mathbf{s}+\eta \tau\left|U-\overrightarrow{\mathbf{e}}_{r}(\theta)\right|, \theta\right) G_{\eta}\left(\mathbf{s}+\eta \tau\left|U-\overrightarrow{\mathbf{e}}_{r}(\theta)\right|, \theta\right)}{\left|\Psi_{\eta}\left(\mathbf{s}+\tau \eta\left|U-\overrightarrow{\mathbf{e}}_{r}(\theta)\right|, \overrightarrow{\mathbf{e}}_{r}(\theta)\right)-\Psi_{\eta}(\mathbf{s}, U)\right|^{3}} \\
& =\frac{\left|U-\overrightarrow{\mathbf{e}}_{r}(\theta)\right| \chi(\mathbf{s})\left(U-\overrightarrow{\mathbf{e}}_{r}(\theta)\right) A\left(\mathbf{s}+\eta \tau\left|U-\overrightarrow{\mathbf{e}}_{r}(\theta)\right|, \theta\right) G_{\eta}\left(\mathbf{s}+\eta \tau\left|U-\overrightarrow{\mathbf{e}}_{r}(\theta)\right|, \theta\right)}{|\tau| U-\overrightarrow{\mathbf{e}}_{r}(\theta)\left|\Lambda\left(\mathbf{s}, \mathbf{s}+\tau \eta\left|U-\overrightarrow{\mathbf{e}}_{r}(\theta)\right|\right)+\chi\left(\mathbf{s}+\tau \eta\left|U-\overrightarrow{\mathbf{e}}_{r}(\theta)\right|\right)\left(\overrightarrow{\mathbf{e}}_{r}(\theta)\right)-\chi(\mathbf{s})(U)\right|^{3}} .
\end{aligned}
$$

Hence, using Lemma 4.1, and since $A$ and $G_{\eta}$ are uniformly bounded, then there exists a constant $M$ independent of $\eta, \mathbf{s}, U, \theta$, and $\tau$ such that :

$$
\left|K_{\eta}(\mathbf{s}, U, \theta, \tau)\right| \leq M \frac{\eta^{3}\left|U-\overrightarrow{\mathbf{e}}_{r}(\theta)\right|^{2}}{\left(\left|\tau^{2} \eta^{2}\right| U-\left.\overrightarrow{\mathbf{e}}_{r}(\theta)\right|^{2}+\eta^{2}\left|U-\overrightarrow{\mathbf{e}}_{r}(\theta)\right|^{2}\right)^{\frac{3}{2}}} \leq \frac{M}{\left|U-\overrightarrow{\mathbf{e}}_{r}(\theta)\right|} \frac{1}{\left(1+\tau^{2}\right)^{\frac{3}{2}}} .
$$

Therefore, when $\eta$ tends to zero, for a fixed $(\mathbf{s}, U, \theta, \tau), K_{\eta}(\mathbf{s}, U, \theta, \tau)$ tends to

$$
K_{0}(\mathbf{s}, U, \theta, \tau):=\frac{\chi(\mathbf{s})\left(U-\overrightarrow{\mathbf{e}}_{r}(\theta)\right) A(\mathbf{s}, \theta)}{\left(\left|U-\overrightarrow{\mathbf{e}}_{r}(\theta)\right|^{2} \tau^{2}+\left|\chi(\mathbf{s})\left(U-\overrightarrow{\mathbf{e}}_{r}(\theta)\right)\right|^{2}\right)^{\frac{3}{2}}} G_{0}(\mathbf{s}, \theta),
$$

since $\Lambda(\mathbf{s}, \mathbf{s})=\Gamma^{\prime}(\mathbf{s})$ is orthogonal to the range of $\chi(\mathbf{s})$. 
With Estimate (4.4), we obtain by the Lebesgue dominated convergence theorem that for all $(\mathbf{s}, U)$, $I_{4,2}^{\eta}(\mathbf{s}, U)$ tends to

$$
D(\mathbf{s}, U)=\frac{1}{4 \pi} \int_{0}^{2 \pi} \int_{\mathbb{R}} K_{0}(\mathbf{s}, U, \theta, \tau) d \tau d \theta=\frac{1}{2 \pi} \int_{0}^{2 \pi} \frac{\chi(\mathbf{s})\left(U-\overrightarrow{\mathbf{e}}_{r}(\theta)\right) A(\mathbf{s}, \theta)}{\left|\chi(\mathbf{s})\left(U-\overrightarrow{\mathbf{e}}_{r}(\theta)\right)\right|^{2}} G_{0}(\mathbf{s}, \theta) d \theta .
$$

In addition, using Estimate (4.4) we obtain that for all $\eta$, and for all $(\mathbf{s}, U) \in \mathcal{O}$,

$$
\left|I_{4,2}^{\eta}(\mathbf{s}, U)\right| \leq \frac{1}{2 \pi} \int_{0}^{2 \pi} \frac{M}{\left|U-\overrightarrow{\mathbf{e}}_{r}(\theta)\right|} d \theta .
$$

Using that $U=r e^{i \zeta}$, such that $(\zeta, r) \in[0,2 \pi] \times B(0,1)$ and by changing the variable $v=\theta-\zeta$ we obtain

$$
\int_{0}^{2 \pi} \frac{M}{\left|U-\overrightarrow{\mathbf{e}}_{r}(\theta)\right|} d \theta=\int_{0}^{2 \pi} \frac{M}{\left|r-e^{i(\theta-\zeta)}\right|} d \theta=\int_{-\zeta}^{2 \pi-\zeta} \frac{M}{\left|r-e^{i v}\right|} d v .
$$

Since the function $v \rightarrow r-e^{i v}$ is $2 \pi$-periodic, we get:

$$
\int_{0}^{2 \pi} \frac{M}{\left|U-\overrightarrow{\mathbf{e}}_{r}(\theta)\right|} d \theta=\int_{0}^{2 \pi} \frac{M}{\sqrt{(r-\cos \theta)^{2}+\sin ^{2} \theta}} d \theta .
$$

In order to show the right hand of $(4.5)$ is in $L^{2}(\mathcal{O})$, we have the following Lemma.

Lemma 4.2. There exists a constant $C>0$, such that for all $(\theta, r) \in[0,2 \pi] \times B(0,1)$, we have

$$
(r-\cos \theta)^{2}+\sin ^{2} \theta \geq C\left((r-1)^{2}+\theta^{2}\right) .
$$

Proof of Lemma 4.2 Denoting by $g(\theta, r)=(r-\cos \theta)^{2}+\sin ^{2} \theta$, note that

$$
g(0,1)=0, \quad \nabla g(0,1)=0 \quad \text { and Hess } g(0,1)=\left(\begin{array}{ll}
2 & 0 \\
0 & 2
\end{array}\right) .
$$

Then by Taylor expansion in the neighbourhood of $(0,1)$, so that for all $(\theta, r) \in B((0,1), \nu)$

$$
g(\theta, r)=\int_{0}^{1}(\theta, r-1)^{T} \operatorname{Hess} g((0,1)+t(\theta, r-1))(\theta, r-1)(1-t) d t .
$$

Since the Hess $g(0,1)$ is strictely positive, there exists $C_{1}>0$, such that

$$
g(\theta, r) \geq C_{1}\left(\theta^{2}+(r-1)^{2}\right) .
$$

We remark that the function $\frac{g(\theta, r)}{\left(\theta^{2}+(r-1)^{2}\right)}$ is continuous and positive, furthermore $[0,2 \pi] \times \overline{B(0,1)}-$ $B((0,1), \nu)$ is compact, so there exists $C_{2}$ such that

$$
\frac{g(\theta, r)}{\left(\theta^{2}+(r-1)^{2}\right)} \geq C_{2}
$$

taking $C=\min \left\{C_{1}, C_{2}\right\}$, we conclude the proof of our Lemma.

Now, using the above Lemma, we obtain:

$$
\int_{0}^{2 \pi} \frac{M}{\left|U-\overrightarrow{\mathbf{e}}_{r}(\theta)\right|} d \theta \leq \int_{0}^{2 \pi} \frac{M}{\sqrt{(r-1)^{2}+\theta^{2}}} d \theta .
$$

Using the change of variable $u=\frac{\theta}{|r-1|}$, yields:

$$
\int_{0}^{2 \pi} \frac{1}{\sqrt{(r-1)^{2}+\theta^{2}}} d \theta \leq \int_{0}^{\frac{2 \pi}{|a-1|}} \frac{1}{\sqrt{1+u^{2}}} d u \leq \int_{0}^{1} d u+\int_{1}^{\mid \frac{2 \pi}{|a-1|}} \frac{1}{u} d u .
$$


Then, we conclude that

$$
\int_{0}^{2 \pi} \frac{M}{\left|U-\overrightarrow{\mathbf{e}}_{r}(\theta)\right|} d \theta \leq M\left(1+\log \left(\frac{2 \pi}{|r-1|}\right)\right) \in L^{2}(\mathcal{O}) .
$$

Furthermore $\left|I_{4,2}^{\eta}\right|$ strongly tends to $D(\mathbf{s}, U)$ in $L^{2}(\mathcal{O})$.

We denote $X=u \mathbf{a}(\mathbf{s})+i v \mathbf{b}(\mathbf{s})$. We remark that $\cos \theta=\frac{1}{2}\left(z+\frac{1}{z}\right)$ and $\sin \theta=\frac{1}{2 i}\left(z-\frac{1}{z}\right)$, with $z=e^{i \theta}$. So $D(\mathbf{s}, U)$ can be written as an integral of a meromorphic function $F$ on the circle $\mathcal{C}(0,1)$ of center 0 and radius 1 :

$$
D(\mathbf{s}, U)=\frac{1}{2 i \pi} \int_{\mathcal{C}(0,1)} F(z) d z
$$

with

$$
F(z)=\frac{\left(\mathbf{a}(\mathbf{s}) w_{2}(\mathbf{s})+i \mathbf{b}(\mathbf{s}) w_{3}(\mathbf{s})\right) z^{2}+\mathbf{a}(\mathbf{s}) w_{2}(\mathbf{s})+i w_{3}(\mathbf{s}) \mathbf{b}(s)}{z\left((\mathbf{b}(\mathbf{s})-\mathbf{a}(\mathbf{s})) z^{2}+2 \bar{X} z-(\mathbf{a}(\mathbf{s})+\mathbf{b}(\mathbf{s}))\right)} .
$$

In [9], using complex analysis arguments, Jizzini proves the following proposition:

Proposition 4.2. The meromorphic function $F$ has only one pole $z=0$ and its residue is given by:

$$
\operatorname{Res}_{F}(0)=\frac{\mathbf{b}(\mathbf{s}) w_{2}(\mathbf{s})+i w_{3}(\mathbf{s}) \mathbf{a}(\mathbf{s})}{-(\mathbf{a}(s)+\mathbf{b}(\mathbf{s}))}
$$

For the convenience of the reader, we reproduce here the proof of the proposition (see [9]).

Proof of Proposition 4.2. Let us suppose that $z \neq 0$ is a pole of $F$ inside $C(0,1)$ which means $z$ verifies the following equation

$$
(\mathbf{b}(\mathbf{s})-\mathbf{a}(\mathbf{s})) z^{2}+2 \bar{X} z-(\mathbf{a}(\mathbf{s})+\mathbf{b}(\mathbf{s}))=0 .
$$

Denoting $\delta(\mathbf{s})=\frac{\mathbf{b}(\mathbf{s})}{\mathbf{a}(\mathbf{s})}$, we can rewrite (4.6) as follows:

$$
(\delta(\mathbf{s})-1) z+\frac{\bar{X}}{\mathbf{a}(\mathbf{s})}-(\delta(\mathbf{s})+1) \frac{1}{z}=0 .
$$

Since $X$ is inside the ellipse $E(\mathbf{a}(\mathbf{s}), \delta(\mathbf{s}) \mathbf{a}(\mathbf{s}))$, there exists $\lambda_{0}(\mathbf{s}) \in\left[0,1\left[\right.\right.$ and $\left.\theta_{0}(\mathbf{s}) \in\right]-\pi, \pi[$ such that

$$
\frac{\bar{X}}{\mathbf{a}(\mathbf{s})}=\lambda_{0} \cos \theta_{0}(\mathbf{s})+i \lambda_{0} \delta(\mathbf{s}) \sin \theta_{0}(\mathbf{s}) \text {. }
$$

Thus the equation (4.7) is equivalent to the following equation

$$
(\delta(\mathbf{s})-1) z+\lambda_{0} z_{0}(1-\delta(\mathbf{s}))+\frac{\lambda_{0}}{z_{0}}(1+\delta(\mathbf{s}))-(1+\delta(\mathbf{s})) \frac{1}{z}=0
$$

where $z_{0}=e^{i \theta_{0}}$. By simple calculation we obtain that

$$
\frac{1}{z}-\frac{\lambda_{0}}{z_{0}}=\frac{\delta(\mathbf{s})-1}{\delta(\mathbf{s})+1}\left(z-\lambda_{0} z_{0}\right)
$$

Furthermore since $z$ is a pole in $B_{2}(0,1)$ and $\left|\frac{\delta-1}{\delta+1}\right|<1$, we obtain

$$
\left|\frac{1}{z}-\frac{\lambda_{0}}{z_{0}}\right|<\left|z-\lambda_{0} z_{0}\right|
$$

So, we get

$$
\left|1-\lambda_{0} \varsigma\right|<\left|\varsigma-\lambda_{0}\right|
$$

where $\varsigma=\frac{z}{z_{0}}$, which means

$$
1-|\varsigma|^{2}<\lambda_{0}\left(1-|\varsigma|^{2}\right)
$$


Hence, we conclude that $\lambda_{0}>1$ which is a contradiction. Thus $F$ has one simple pole $z=0$ and by direct application of the residue Theorem, we conclude the proof of Proposition 4.2.

Finally we conclude that:

$$
D(\mathbf{s}, U)=\frac{1}{2 i \pi} \int_{\mathcal{C}(0,1)} F(z) d z=-\frac{\mathbf{b}(\mathbf{s})}{\mathbf{a}(\mathbf{s})+\mathbf{b}(\mathbf{s})} w_{2}(\mathbf{s})-\frac{\mathbf{a}(\mathbf{s})}{\mathbf{a}(\mathbf{s})+\mathbf{b}(\mathbf{s})} w_{3}(\mathbf{s}) .
$$

This ends the proof of Proposition 4.1.

[7] [2]

\section{References}

[1] D. A. Allwood, G. Xiong, C. C. Faulkner, D. Atkinson, D. Petit, and R. P. Cowburn. Magnetic domain-wall logic. Science, 309(5741):1688-1692, 2005.

[2] F. Alouges and A. Soyeur. On global weak solutions for landau-lifshitz equations: Existence and nonuniqueness. Nonlinear Analysis: Theory, Methods 83 Applications, 18(11):1071 - 1084, 1992.

[3] G. Bonithon. Landau-Lifschitz-Gilbert equation with applied electric current. In The 6th AIMS Conference on Dynamical Systems, Differential Equations and Applications, pages 138-144, Poitiers, France, June 2006.

[4] O. Boulle, G. Malinowski, and M. Klui. Current-induced domain wall motion in nanoscale ferromagnetic elements. Materials Science and Engineering: R: Reports, 72(9):159 - 187, 2011.

[5] W. F. Brown. Micromagnetics. John Willey and Sons, New York, 1963.

[6] G. Carbou and S. Labbé. Stabilization of walls for nano-wires of finite length. ESAIM: Control, Optimisation and Calculus of Variations, 18(1):121, 2012.

[7] K. Chacouche and R. Hadiji. Ferromagnetic of nanowires of infinite length and infinite thin films. Zeitschrift für angewandte Mathematik und Physik, 66(6):3519-3534, Dec 2015.

[8] Y. Gaididei, A. Goussev, V. P. Kravchuk, O. V. Pylypovskyi, J. M. Robbins, D. D. Sheka, V. Slastikov, and S. Vasylkevych. Magnetization in narrow ribbons: curvature effects. Journal of Physics A: Mathematical and Theoretical, 50(38):385401, 2017.

[9] R. Jizzini. Etude mathématique d'un modèle de fil ferromagnétique en présence d'un courant électrique. $\mathrm{PhD}$ thesis, 2013. Thèse de doctorat dirigée par Carbou, Gilles Mathmatiques appliquées Bordeaux 12013.

[10] L. D. Landau and E. M. Lifshitz. Electrodynamique des milieux continus. Physique théorique. MIR, Moscow, 1969.

[11] S. S. P. Parkin, M. Hayashi, and L. Thomas. Magnetic domain-wall racetrack memory. Science, 320(5873):190-194, 2008.

[12] O. V. Pylypovskyi, D. D. Sheka, V. P. Kravchuk, K. V. Yershov, D. Makarov, and Y. Gaididei. Rashba torque driven domain wall motion in magnetic helices. Scientific Reports, 6:23316 EP -, 032016 .

[13] D. D. Sheka, V. P. Kravchuk, K. V. Yershov, and Y. Gaididei. Torsion-induced effects in magnetic nanowires. Phys. Rev. B, 92:054417, Aug 2015.

[14] D. M. Silevitch, M. Tanase, C. L. Chien, and D. H. Reich. Room temperature domain wall pinning in bent ferromagnetic nanowires. eprint arXiv:cond-mat/0308579, Aug. 2003. 
[15] V. Slastikov and C. Sonnenberg. Reduced models for ferromagnetic nanowires. IMA Journal of Applied Mathematics, 77(2):220-235, 42012.

[16] M. Tanase, D. M. Silevitch, C. L. Chien, and D. H. Reich. Magnetotransport properties of bent ferromagnetic nanowires. Journal of Applied Physics, 93(10):7616-7618, 2003.

[17] A. Thiaville, Y. Nakatani, J. Miltat, and Y. Suzuki. Micromagnetic understanding of currentdriven domain wall motion in patterned nanowires. EPL (Europhysics Letters), 69(6):990, 2005.

[18] K. V. Yershov, V. P. Kravchuk, D. D. Sheka, and Y. Gaididei. Curvature-induced domain wall pinning. prb, 92(10):104412, Sept. 2015.

[19] K. V. Yershov, V. P. Kravchuk, D. D. Sheka, and Y. Gaididei. Curvature and torsion effects in spin-current driven domain wall motion. Phys. Rev. B, 93:094418, Mar 2016. 